\title{
Visible actions on irreducible multiplicity-free spaces
}

\author{
By Atsumu SASAKI \\ Department of Mathematical Sciences, School of Science and Engineering, Waseda University, \\ 3-4-1 Ohkubo, Shinjuku-ku, Tokyo 169-8555, Japan \\ (Communicated by Shigefumi MorI, M.J.A., Sept. 12, 2007)
}

\begin{abstract}
A holomorphic action of a Lie group $G$ on a complex manifold $D$ is called strongly visible if there exist a totally real submanifold $S$ which meets every $G$-orbit in $D$ and an anti-holomorphic diffeomorphism $\sigma$ such that $\left.\sigma\right|_{S}=\operatorname{id}_{S}$ and $\sigma$ preserves every $G$-orbit. In this paper, we prove that Kac's multiplicity-free space is strongly visible, that is, if $\left(G_{\mathbf{C}}, V\right)$ is an irreducible multiplicity-free space of a complex reductive Lie group $G_{\mathbf{C}}$, then a compact real form of $G_{\mathrm{C}}$ acts on $V$ in a strongly visible fashion. Furthermore, we give an explicit description of the choice of a totally real submanifold $S$ and an anti-holomorphic involution $\sigma$. This gives an evidence to Kobayashi's conjecture [8, Conjecture 3.2], that is, $\operatorname{dim}_{\mathbf{R}} S$ coincides with the rank of the polynomial representation of $G_{\mathbf{C}}$ on $\mathbf{C}[V]$ in this setting.
\end{abstract}

Key words: Complex manifold; (strongly) visible action; totally real submanifold; multiplicity-free space; multiplicity-free representation.

Suppose a Lie group $G$ acts holomorphically on a complex manifold $D$.

Definition 1. This action is strongly visible if the following two conditions hold:

(a) There exists a real submanifold $S$ (which we call a slice) such that

$$
D=G \cdot S,
$$

that is, $S$ meets every $G$-orbit in $D$.

(b) There exists an anti-holomorphic diffeomorphism $\sigma$ on $D$ such that

$$
\left.\sigma\right|_{S}=\operatorname{id}_{S}
$$

$$
\sigma \text { preserves each } G \text {-orbit in } D \text {. }
$$

We note that the submanifold $S$ is automatically totally real, that is, $T_{x} S \cap J_{x}\left(T_{x} S\right)=\{0\}$ holds for any $x \in S$ by the condition (b).

The notion of (strongly) visible actions has been introduced by Kobayashi in [6] as a basic assumption for the propagation theorem of multiplicity-free property from fibers to the space of holomorphic sections, where the submanifold $S$ plays an important role (see $[7,9]$ ). To be more precise, Definition 1 is slightly stronger than the original definition of strongly visible actions in

2000 Mathematics Subject Classification. Primary 32M05; Secondary 22E46, 20G05, 32M15.
$[7,9]$ in the sense that the original definition of strongly visible actions can be verified locally in a $G$-invariant open subset.

The notion of (strongly) visible actions is also interesting for its own from geometric viewpoints, as one of the three relevant notions: polar actions in Riemannian geometries (e.g. Podestà-Thorbergsson), coisotropic actions in symplectic geometries (Guillemin-Sternberg, Huckleberry-Wurzbacher), and visible actions in complex geometries (Kobayashi) (see $[7, \S 4]$ for recent progress in this direction).

Another interesting aspect of (strongly) visible actions is that visible actions naturally bring us to various decomposition theorems of Lie groups and homogeneous spaces. For instance, consider the linear fractional action of $G=S L(2, \mathbf{R})$ on a complex upper half plane $\mathcal{H}_{+}$. Then the Iwasawa decomposition $G=N A K$ explains that the action of a maximal unipotent subgroup $N$ on $\mathcal{H}_{+}$is strongly visible, whereas the Cartan decomposition $G=K A K$ explains that the action of a maximal compact subgroup $K$ on $\mathcal{H}_{+}$is also strongly visible (see [7, Figures 5.4 .1 (a), (c)]). Conversely, we may expect that new examples of (strongly) visible actions could reveal new decomposition theorems of Lie groups and homogeneous spaces. In this sense, finding an explicit description of a totally real submanifold $S$ in Definition 1 becomes a guiding principle to find decomposition theorems. 
Originally, the notion of (strongly) visible actions was introduced to give an unified explanation of various multiplicity-free theorems $[6,7]$. Conversely if we are given multiplicity-free representations, we could expect that (some real forms of) the group acts on the underlying geometry in a (strongly) visible fashion (e.g. $[10,11]$ ).

In this paper, we consider multiplicity-free spaces in the sense of Kac [4]. Given an algebraic representation $\pi: G_{\mathbf{C}} \rightarrow G L_{\mathbf{C}}(V)$ of a connected complex reductive Lie group $G_{\mathbf{C}}$ on a finite dimensional complex vector space $V$, we have a representation of $G_{\mathbf{C}}$ on the polynomial ring $\mathbf{C}[V]$. We say $V$ is a mutiplicity-free space of $G_{\mathbf{C}}$ (or simply, $\left(G_{\mathbf{C}}, V\right.$ ) is a multiplicity-free space) if $\mathbf{C}[V]$ decomposes into the multiplicity-free sum of irreducible representations of $G_{\mathbf{C}}$.

We write the irreducible decomposition of $\mathbf{C}[V]$ as follows:

$$
\mathbf{C}[V] \simeq \bigoplus_{\lambda \in \Lambda} P_{\lambda} .
$$

Here, $P_{\lambda}$ is an irreducible representation of $G_{\mathbf{C}}$ with a highest weight $\lambda$. Then the set of highest weights $\Lambda$ forms a semigroup, and there exist linearly independent highest weights $\lambda_{1}, \ldots, \lambda_{k} \in \Lambda$ such that $\Lambda=\left\{r_{1} \lambda_{1}+\cdots+r_{k} \lambda_{k}: r_{1}, \ldots, r_{k} \in \mathbf{Z}_{\geq 0}\right\}$ $([2,5])$. We say the rank $k$ of the semigroup $\Lambda$ is the rank of the polynomial representation $\mathbf{C}[V]$ (or the number of fundamental generators), which was computed in Howe and Umeda [3, Table 15.1].

The multiplcity-free space $\left(G_{\mathbf{C}}, V\right)$ is irreducible if $\pi$ is irreducible. The classification of irreducible multiplicity-free spaces was accomplished by Kac [4, Theorem 3] under the assumption that $G_{\mathbf{C}}$ is reductive (see also [2] for a survey). According to the classification, $G_{\mathbf{C}}$ is of the form $H_{\mathbf{C}} \times \mathbf{C}^{*}$ or $H_{\mathbf{C}}$ where $H_{\mathbf{C}}$ is a semisimple complex Lie group. We recall his list in a way that fits into our framework of visible actions.

Since $G_{\mathrm{C}}$ is reductive, there exists a compact real form of $G_{\mathbf{C}}$, denoted by $G_{U}$. By Weyl's unitary trick, the category of holomorphic representations $\left(G_{\mathrm{C}}, V\right)$ is equivalent to that of complex representations $\left(G_{U}, V\right)$. So we give Kac's classification of irreducible multiplicity-free spaces in terms of $\left(G_{U}, V\right)$ in Table I. Here are some conditions on integers $m$ and $n$ in Table I. In (1a) $n \geq 2$; in (3) $n \geq 3$; in (6a) $m \neq n$; in (7) $n \geq 2$; in (8) $n \geq 2$; in (9a) $m \geq 5$. In (13) and (14), $G_{2}$ and $E_{6}$ denote the
Table I. Irreducible multiplicity-free spaces

\begin{tabular}{c|c|c|c}
\hline & \multicolumn{2}{|c|}{ Kac's list } & Slice \\
\hline & $G_{U}$ & $V$ & $\operatorname{dim} \mathbf{R} S$ \\
$1 \mathrm{a}$ & $S U(n)$ & $\mathbf{C}^{n}$ & 1 \\
$1 \mathrm{~b}$ & $\mathbf{T}$ & $\mathbf{C}$ & \\
2 & $S p(n)$ & $\mathbf{C}^{2 n}$ & 1 \\
3 & $S O(n) \times \mathbf{T}$ & $\mathbf{C}^{n}$ & 2 \\
4 & $S U(n) \times \mathbf{T}$ & $S^{2}\left(\mathbf{C}^{n}\right)$ & $n$ \\
$5 \mathrm{a}$ & $S U(2 n+1)$ & $\bigwedge^{2}\left(\mathbf{C}^{2 n+1}\right)$ & $n$ \\
$5 \mathrm{~b}$ & $S U(2 n) \times \mathbf{T}$ & $\bigwedge^{2}\left(\mathbf{C}^{2 n}\right)$ & \\
$6 \mathrm{a}$ & $S U(m) \times S U(n)$ & $\mathbf{C}^{m} \otimes \mathbf{C}^{n}$ & $\min (m, n)$ \\
$6 \mathrm{~b}$ & $S U(n) \times S U(n) \times \mathbf{T}$ & $\mathbf{C}^{n} \otimes \mathbf{C}^{n}$ & \\
7 & $S U(2) \times S p(n) \times \mathbf{T}$ & $\mathbf{C}^{2} \otimes \mathbf{C}^{2 n}$ & 3 \\
8 & $S U(3) \times S p(n) \times \mathbf{T}$ & $\mathbf{C}^{3} \otimes \mathbf{C}^{2 n}$ & $5(n=2)$ \\
& & & $6(n \geq 3)$ \\
$9 \mathrm{a}$ & $S U(m) \times S p(2)$ & $\mathbf{C}^{m} \otimes \mathbf{C}^{4}$ & 6 \\
$9 \mathrm{~b}$ & $S U(4) \times S p(2) \times \mathbf{T}$ & $\mathbf{C}^{4} \otimes \mathbf{C}^{4}$ & \\
10 & $S p i n(7) \times \mathbf{T}$ & $\mathbf{C}^{8}$ & 2 \\
11 & $S p i n(9) \times \mathbf{T}$ & $\mathbf{C}^{16}$ & 3 \\
12 & $S p i n(10)$ & $\mathbf{C}^{16}$ & 2 \\
13 & $G 2 \times \mathbf{T}$ & $\mathbf{C}^{7}$ & 2 \\
14 & $E_{6} \times \mathbf{T}$ & $\mathbf{C}^{27}$ & 3 \\
\hline
\end{tabular}

corresponding simply connected Lie groups of exceptional type. Since local isomorphisms of $G_{U}$ are not the main issue here, we shall use the global form of $G_{U}$ as in Table I.

Remark 2. In (1a), (2), (5a), (6a), (9a) and (12), $G_{U}$ is semisimple. We note that $\left(G_{U} \times \mathbf{T}, V\right)$ also give irreducible multiplicity-free spaces in these cases (see [4, Theorem 3]) but we have omitted there trivial cases in Table I.

Our main theorem is stated as follows:

Theorem A. Let $\left(G_{\mathbf{C}}, V\right)$ be an irreducible multiplicity-free space, and $G_{U}$ a compact real form of $G_{\mathbf{C}}$.

(1) The action of $G_{U}$ on $V$ is strongly visible.

(2) We can take a slice $S$ (see Definition 1) such that $\operatorname{dim}_{\mathbf{R}} S$ is equal to the rank of the polynomial representation $\mathbf{C}[V]$.

The dimension of our slice $S$ is listed in the right column of Table I.

Combining Theorem A with [7, Theorem 5] (or [9, Corollary 2.4]), we obtain the following corollary immediately.

Corollary B. Let $\pi: G_{\mathbf{C}} \rightarrow G L_{\mathbf{C}}(V)$ be an irreducible algebraic representation of a connected complex reductive Lie group $G_{\mathbf{C}}$ on a finite dimensional complex vector space $V$. Then the following 
two conditions are equivalent:

(i) $V$ is a multiplicity-free space of $G_{\mathbf{C}}$.

(ii) The action of a compact real form $G_{U}$ of $G_{\mathbf{C}}$ on $V$ is strongly visible in the sense of Definition 1 . The rest of this paper is devoted to the proof of Theorem A. For the cases discussed in Remark 2, the orbit of $G_{U} \times \mathbf{T}$ on $V$ coincides with that of $G_{U}$ on $V$. Therefore Theorem A for $G_{U} \times \mathbf{T}$ implies Theorem A for $G_{U}$ and vice versa. Thus, we may and do assume that $G_{U}$ always contains one dimensional center T. Now we divide irreducible multiplicity-free spaces into the following three cases.

Case 1. $\left(G_{U}, V\right)$ is in cases $(1),(3),(4),(5)$, (6), (12) and (14).

Case 2. $\left(G_{U}, V\right)$ is in cases (7), (8) and (9), that is, of the form $\left(S U(m) \times S p(n) \times \mathbf{T}, \mathbf{C}^{m} \otimes\right.$ $\left.\mathbf{C}^{2 n}\right)$.

Case 3. We divide the remaining cases (2), (10), (11) and (13) into the following subcases.

(3-a) $\left(G_{U}, V\right)=\left(S p(n) \times \mathbf{T}, \mathbf{C}^{2 n}\right)$.

$(3-\mathrm{b})\left(G_{U}, V\right)=\left(\operatorname{Spin}(7) \times \mathbf{T}, \mathbf{C}^{8}\right)$ and $\left(G_{2} \times \mathbf{T}, \mathbf{C}^{7}\right)$. $(3-\mathrm{c})\left(G_{U}, V\right)=\left(\operatorname{Spin}(9) \times \mathbf{T}, \mathbf{C}^{16}\right)$.

First, we consider Case 1.

Proof of Theorem A in Case 1. We shall see below that the proof of Theorem A in this case reduces to a special case of [11, Theorem 1.5].

Suppose $\left(G_{U}, V\right)$ is in Case 1 . Then there exists a non-compact, simply connected simple Lie group $G$ of Hermitian type such that a maximal compact subgroup $K$ of $G$ is isomorphic to $G_{U}$ and the induced action of the adjoint representation $(K, \mathfrak{g} / \mathfrak{k})$ is isomorphic to the given representation $\left(G_{U}, V\right)$ (up to the action of the center of $G_{U}$ ). Here $\mathfrak{g}$ is the Lie algebra of $G$ and $\mathfrak{k}$ is that of $K$. On the other hand, it is proved in [11, Theorem 1.5] that the $K$-action on the Hermitian symmetric space $G / K$ is strongly visible. Let $\mathfrak{g}=\mathfrak{k}+\mathfrak{p}$ be the corresponding Cartan decomposition of Lie algebra $\mathfrak{g}$. Since $K$ acts on $\mathfrak{p}$ as an adjoint representation and $G / K$ is realized as a bounded symmetric domain in $\mathfrak{p}$, we can show similarly that the $K$ action on $\mathfrak{p}$ is strongly visible with a choice of slice $S$ such that $S$ is a maximal abelian subspace $\mathfrak{a}$ in $\mathfrak{p}$. In particular $\operatorname{dim}_{\mathbf{R}} S=\operatorname{dim} \mathfrak{a}=\mathbf{R}$-rank $G / K$.

We will call $\left(G_{U}, V\right)$ in Case 1 of "Hermitian type".

Next, we consider Case 2 . We identify $S U(m) \times S p(n) \times \mathbf{T} \simeq U(m) \times S p(n) \quad$ and $\quad \mathbf{C}^{m} \otimes$ $\mathbf{C}^{2 n} \simeq M(m, 2 n ; \mathbf{C})$, the vector space consisting of $(m \times 2 n)$-complex matrices. Here we let $U(m) \times$
$S p(n)$ act on $M(m, 2 n ; \mathbf{C})$ by

$$
(g, h) \cdot X=g X h^{-1}(g \in U(m), h \in S p(n))
$$

where we realize $S p(n)$ in $U(2 n)$ in a standard way.

Proof of Theorem A in Case 2. Case 2 consists of three cases (7), (8) and (9), for which the proof of Theorem A can be given similarly. Here, we consider only the case $(7)$, namely, $\left(G_{U}, V\right)=$ $(U(2) \times S p(n), M(2,2 n ; \mathbf{C}))$, which is simplest among the three cases. Let $\vec{e}_{1}, \ldots, \vec{e}_{2 n}$ be the standard basis of $\mathbf{C}^{2 n}$. We write an element $X \in V$ as

$$
X=\left(\begin{array}{ccc}
x_{1} & \cdots & x_{2 n} \\
y_{1} & \cdots & y_{2 n}
\end{array}\right)=\left(\begin{array}{c}
{ }^{t} \vec{x} \\
{ }^{t} \vec{y}
\end{array}\right) .
$$

By using the first factor $U(2)$ of $G_{U}$, we can transform $X$ such that $(\vec{x}, \vec{y})=0$ where $(\cdot, \cdot)$ denotes the standard Hermitian inner product on $\mathbf{C}^{2 n}$.

Second, we take $h_{1} \in S p(n)$ such that ${ }^{t} \vec{x} h_{1}^{-1}=$ $r_{1}^{t} \vec{e}_{1}$ for $r_{1}=\left(\left|x_{1}\right|^{2}+\cdots+\left|x_{2 n}\right|^{2}\right)^{1 / 2}$. Let

$$
\left(\begin{array}{c}
{ }^{t} \overrightarrow{x^{\prime}} \\
{ }^{t} \overrightarrow{y^{\prime}}
\end{array}\right):=\left(\begin{array}{c}
{ }^{t} \vec{x} h_{1}^{-1} \\
{ }^{t} \vec{y} h_{1}^{-1}
\end{array}\right)=\left(\begin{array}{cccc}
r_{1} & 0 & \cdots & 0 \\
y_{1}^{\prime} & y_{2}^{\prime} & \cdots & y_{2 n}^{\prime}
\end{array}\right) .
$$

Since $\left(\overrightarrow{x^{\prime}}, \overrightarrow{y^{\prime}}\right)=(\vec{x}, \vec{y})=0$, we get $y_{1}^{\prime}=0$. Thus $X h_{1}^{-1}$ is of the form

$$
X h_{1}^{-1}=\left(\begin{array}{ccccc}
r_{1} & 0 & 0 & \cdots & 0 \\
0 & y_{2}^{\prime} & y_{3}^{\prime} & \cdots & y_{2 n}^{\prime}
\end{array}\right) .
$$

Third, we write

$$
y_{2}^{\prime}=r_{2} e^{\sqrt{-1} \theta}\left(r_{2} \geq 0, \theta \in \mathbf{R} / 2 \pi \mathbf{Z}\right)
$$

and take $h_{2} \in S p(n-1)$ such that

$$
e^{-\sqrt{-1} \theta}\left(y_{3}^{\prime}, \ldots, y_{2 n}^{\prime}\right) h_{2}^{-1}=r_{3}(1,0, \ldots, 0)
$$

for $r_{3}=\left(\left|y_{3}^{\prime}\right|^{2}+\cdots+\left|y_{2 n}^{\prime}\right|^{2}\right)^{1 / 2}$. We regard $S p(n-1)$ as a subgroup of $S p(n)$ and set $g_{1}:=\operatorname{diag}(1$, $\left.e^{-\sqrt{-1} \theta}\right) \in U(2)$. Then

$$
\begin{aligned}
g_{1}\left(X h_{1}^{-1}\right) h_{2}^{-1} & =\left(\begin{array}{cccccc}
r_{1} & 0 & 0 & 0 & \cdots & 0 \\
0 & r_{2} & r_{3} & 0 & \cdots & 0
\end{array}\right) \\
& =: X\left(r_{1}, r_{2}, r_{3}\right) .
\end{aligned}
$$

This implies that

$$
S=\left\{X\left(r_{1}, r_{2}, r_{3}\right): r_{1}, r_{2}, r_{3} \in \mathbf{R}\right\}
$$

meets every $G_{U}$-orbit in $V\left(S_{+}:=\left\{X\left(r_{1}, r_{2}, r_{3}\right)\right.\right.$ : $\left.r_{1}, r_{2}, r_{3} \geq 0\right\}$ already holds this property). Now we define an anti-holomorphic involution on $M(2,2 n ; \mathbf{C})$ by $\sigma(X)=\bar{X}(X \in M(2,2 n ; \mathbf{C}))$. Then it is clear that $\left.\sigma\right|_{S}=\operatorname{id}_{S}$ and $\sigma$ preserves each 
$(U(2) \times S p(n))$-orbit in $M(2,2 n ; \mathbf{C})$. Therefore this action is strongly visible with the data $(S, \sigma)$.

Finally, we consider Case 3 . Let $W$ be a real vector space equipped with an inner product $(\cdot, \cdot)_{W}$. We write $S(W)$ for the unit sphere in $W$. Here is an elementary observation:

Lemma 3. If a group $G$ acts linearly on $W$ and acts transitively on $S(W)$, then

$$
W=G \cdot \mathbf{R} v_{0}
$$

for any $v_{0} \in S(W)$.

In addition to Lemma 3, we prepare some more lemmas.

Lemma 4. Retain the setting of Lemma 3. Let $W_{1}=\left(\mathbf{R} v_{0}\right)^{\perp}$ be the orthogonal complementary subspace of $\mathbf{R} v_{0}$ in $W$ We denote by $G_{v_{0}}$ the isotropy subgroup of $G$ at $v_{0}$. If $G_{v_{0}}$ acts transitively on $S\left(W_{1}\right)$, then we have

$$
W \oplus W=G \cdot\left(\mathbf{R} v_{0} \oplus\left(\mathbf{R} v_{0} \oplus \mathbf{R} v_{1}\right)\right)
$$

for any $v_{1} \in S\left(W_{1}\right)$. Here the $G$-action on $W \oplus W$ is given by the diagonal action; $g \cdot\left(w_{1}, w_{2}\right)=$ $\left(g w_{1}, g w_{2}\right)(g \in G)$.

Proof. By the assumption that $G_{v_{0}}$ acts transitively on $S\left(W_{1}\right)$, we obtain $W_{1}=G_{v_{0}} \cdot \mathbf{R} v_{1}$ for any $v_{1} \in S\left(W_{1}\right)$ from Lemma 3 . In view of the decomposition $W=\mathbf{R} v_{0} \oplus W_{1}$, we have

$$
\begin{aligned}
W & =\mathbf{R} v_{0} \oplus\left(G_{v_{0}} \cdot \mathbf{R} v_{1}\right) \\
& =G_{v_{0}} \cdot\left(\mathbf{R} v_{0} \oplus \mathbf{R} v_{1}\right)
\end{aligned}
$$

Hence, Lemma 4 is proved.

Lemma 4 may be regarded as a special case of the following lemma $\left(W_{3}=\{0\}\right)$.

Lemma 5. Retain the setting of Lemma 3 and we write $W_{1}=\left(\mathbf{R} v_{0}\right)^{\perp}$. Assume further that $W_{1}$ decomposes as a direct sum of two vector subspaces $W_{2}$ and $W_{3}$ with the following properties:

(a) $G_{v_{0}}$ acts transitively on $S\left(W_{2}\right)$.

(b) For a fixed $v_{1} \in S\left(W_{2}\right), G_{v_{0}, v_{1}}:=G_{v_{0}} \cap G_{v_{1}}$ acts transitively on $S\left(W_{3}\right)$.

Then we have

$$
W \oplus W=G \cdot\left(\mathbf{R} v_{0} \oplus\left(\mathbf{R} v_{0} \oplus \mathbf{R} v_{1} \oplus \mathbf{R} v_{2}\right)\right)
$$

for any $v_{2} \in S\left(W_{3}\right)$.

Proof. The proof is similar to that of Lemma 4

$\operatorname{Spin}(2 n+1)$ acts on $\mathbf{R}^{2^{n}}$ as a spin representation. $G_{2}$ is an automorphism group $\operatorname{Aut}_{\mathbf{R}}(\mathfrak{C})$, where $\mathfrak{C} \simeq \mathbf{R}^{8}$ stands for the Cayley algebra. Let $e_{0}, \ldots, e_{7}$ be the standard basis of $\mathfrak{C}$, and we set a subspace
Table II. Transitive $G_{U}$-actions on $S(W)$

\begin{tabular}{c|c|c|c|c}
\hline & $G$ & $W$ & $S(W)$ & $G_{v}$ \\
\hline 1 & $S p(n)$ & $\mathbf{C}^{2 n} \simeq \mathbf{R}^{4 n}$ & $S^{4 n-1}$ & $S p(n-1)$ \\
2 & $\operatorname{Spin}(9)$ & $\mathbf{R}^{16}$ & $S^{15}$ & $S p i n(7)$ \\
3 & $\operatorname{Spin}(7)$ & $\mathbf{R}^{8}$ & $S^{7}$ & $G_{2}$ \\
4 & $G_{2}$ & $\mathbf{R}^{7}$ & $S^{6}$ & $S U(3)$ \\
5 & $S U(3)$ & $\mathbf{C}^{3} \simeq \mathbf{R}^{6}$ & $S^{5}$ & $S U(2)$ \\
\hline
\end{tabular}

$\mathfrak{C}_{0}:=\left\{x_{1} e_{1}+\cdots+x_{7} e_{7}: x_{1}, \ldots x_{7} \in \mathbf{R}\right\} \simeq \mathbf{R}^{7}$. Then $\mathfrak{C}_{0}$ is $G_{2}$-invariant, so we define a representation of $G_{2}$ on $\mathfrak{C}_{0}$ (see [1], for example).

Now we recall,

Fact 6. The representation of $G$ on a real vector space $W$ in Table II induces the transitive action on the unit sphere $S(W)$.

The isomorphic class of isotropy subgroups $G_{v}$ is listed in the right column in Table II.

We are ready to prove Theorem A in Case 3.

Proof of Theorem A in Case 3-a. It follows from Fact 6 (1) and Lemma 3 that we have

$$
\mathbf{C}^{2 n}=S p(n) \cdot \mathbf{R} v_{0}
$$

for any $v_{0} \in S^{4 n-1}$. This means that the totally real submanifold $S:=\mathbf{R} v_{0}$ meets every $S p(n)$-orbit in $\mathbf{C}^{2 n}$. As before, let $\vec{e}_{1}, \ldots, \vec{e}_{2 n}$ be the standard basis of $\mathbf{C}^{2 n}$. Now we take $v_{0}=\vec{e}_{1}$. Let $\sigma$ be the standard complex conjugate of $\mathbf{C}^{2 n}$. Then, clearly, $\sigma$ preserves every $S p(n)$-orbit in $\mathbf{C}^{2 n}$. With the data $(S, \sigma)$ the natural action of $S p(n)$ on $\mathbf{C}^{2 n}$ is strongly visible.

Remark 7. The strongly visibility of $S U(n)$ acting on $\mathbf{C}^{n}$ is already proved in Case 1 . An analogous idea to the previous proof of Case 3-a gives an alternative proof of the strongly visibility because $S U(n)$ acts on $S^{2 n-1}$ transitively in $n \geq 2$.

Proof of Theorem A in Case 3-b. We first consider the case $\left(G_{U}, V\right)=\left(G_{2} \times \mathbf{T}, \mathbf{C}^{7}\right)$. The idea of the proof is similar to Case 3 -a, but we need to iterate the argument twice as follows:

Let $G_{2}(\mathbf{C})$ be the complexification of $G_{2}$, and $\mathbf{C}^{7}=\mathbf{R}^{7}+\sqrt{-1} \mathbf{R}^{7} \simeq \mathfrak{C}_{0} \otimes_{\mathbf{R}} \mathbf{C}$.

From Fact 6 (iv) and Lemma 3, we have

$$
\mathbf{R}^{7}=G_{2} \cdot \mathbf{R} v_{0}
$$

for any $v_{0} \in S^{6}$. Since the isotropy subgroup $\left(G_{2}\right)_{v_{0}} \simeq S U(3)$ acts transitively on the complementary subspace $\left(\mathbf{R} v_{0}\right)^{\perp} \simeq \mathbf{R}^{6}$, it follows from Lemma 4 that we have 


$$
\mathbf{C}^{7}=G_{2} \cdot\left(\mathbf{R} v_{0}+\sqrt{-1}\left(\mathbf{R} v_{0}+\mathbf{R} v_{1}\right)\right)
$$

for any $v_{1} \in S^{5}$. Finally, by using the action of the second factor $\mathbf{T}$ of $G_{U}$ on $\mathbf{R}^{7}$, we get

$$
\mathbf{C}^{7}=\left(G_{2} \times \mathbf{T}\right) \cdot\left(\mathbf{R} v_{0}+\sqrt{-1} \mathbf{R} v_{1}\right) .
$$

Thus

$$
S:=\mathbf{R} v_{0}+\sqrt{-1} \mathbf{R} v_{1} \simeq \mathbf{R}^{2}
$$

meets every $G_{U^{-}}$orbit in $\mathbf{C}^{7}$.

We recall $e_{1}, \ldots, e_{7}$ is a basis of $\mathfrak{C}_{0}$, and take $v_{0}=e_{1}, v_{1}=e_{2}$. We define an anti-holomorphic involution $\sigma$ in $\mathbf{C}^{7} \simeq \mathfrak{C}_{0} \otimes_{\mathbf{R}} \mathbf{C}$ by

$$
\sigma\left(c_{1} e_{1}+\cdots+c_{7} e_{7}\right)=\bar{c}_{1} e_{1}-\left(\bar{c}_{2} e_{2}+\cdots+\bar{c}_{7} e_{7}\right)
$$

for $c_{1}, \ldots, c_{7} \in \mathbf{C}$. Then this $\sigma$ preserves each $\left(G_{2} \times \mathbf{T}\right)$-orbit in $\mathbf{C}^{7}$. Therefore, this action is strongly visible with the data $(S, \sigma)$.

The remaining case of Case 3 -b is $\left(G_{U}, V\right)=$ $\left(\operatorname{Spin}(7), \mathbf{C}^{8}\right)$. As $G_{2}$ and its subgroup $S U(3)$ act transitively on $S^{6}$ and $S^{5}$, respectively, in the previous case, we use a fact that $\operatorname{Spin}(7)$ and its subgroup $G_{2}$ act transitively on $S^{7}$ and $S^{6}$, respectively (see Fact 6 ), in this case. Since the proof parallels to the previous case, we omit its proof.

Proof of Theorem A in Case 3-c. This case treats $\left(G_{U}, V\right)=\left(\operatorname{Spin}(9), \mathbf{C}^{16}\right)$.

The proof in this case is similar to Case 3-b. We apply Lemma 5 in place of Lemma 4 . The key ingredient of the proof is that the triple of Lie groups

$$
\operatorname{Spin}(9) \supset \operatorname{Spin}(7) \supset G_{2}
$$

act transitively on the triple of unit spheres

$$
S^{15} \supset S^{7} \supset S^{6},
$$

respectively. Correspondingly, we can take $S$ to be a three dimensional real vector subspace $\mathbf{R}^{3}$ in $\mathbf{C}^{16}$ such that

$$
\mathbf{C}^{16}=(\operatorname{Spin}(9) \times \mathbf{T}) \cdot \mathbf{R}^{3} .
$$

We omit details.

Remark 8. We note that key subgroups in the proof of Cases 3-b and 3-c give rise to non- symmetric spherical varieties

$$
G_{2} / \operatorname{SU}(3), \operatorname{Spin}(7) / G_{2}, \operatorname{Spin}(9) / \operatorname{Spin}(7) .
$$

Finally, the second statement of Theorem A follows by comparing the list of $\operatorname{dim}_{\mathbf{R}} S$ for slices $S$ (see the right column in Table I) with the list of the rank of polynomial representations $\mathbf{C}[V]$ (the number of fundamental generators) given in $[3$, Table 15.1].

\section{References}

[ 1 ] J. F. Adams, Lectures on exceptional Lie groups, Univ. Chicago Press, Chicago, IL, 1996.

[ 2 ] C. Benson and G. Ratcliff, On multiplicity free actions, in Representations of real and p-adic groups, 221-304, Singapore Univ. Press, Singapore, 2004

[ 3 ] R. Howe and T. Umeda, The Capelli identity, the double commutant theorem, and multiplicityfree actions, Math. Ann. 290 (1991), no. 3, 565619.

[ 4 ] V. G. Kac, Some remarks on nilpotent orbits, J. Algebra 64 (1980), no. 1, 190-213.

[ 5 ] F. Knop, Some remarks on multiplicity free spaces, in Representation theories and algebraic geometry (Montreal, PQ, 1997), 301-317, Kluwer Acad. Publ., Dordrecht.

[6] T. Kobayashi, Geometry of multiplicity-free representations of $\mathrm{GL}(n)$, visible actions on flag varieties, and triunity, Acta Appl. Math. 81 (2004), no. 1-3, 129-146.

[ 7 ] T. Kobayashi, Multiplicity-free representations and visible actions on complex manifolds, Publ. Res. Inst. Math. Sci. 41 (2005), no. 3, 497-549.

[8] T. Kobayashi, Introduction to visible actions on complex manifolds and multiplicity-free representations, in Surikaiseki Kokyuroku, 1502 (2006), 82-95, Res. Inst. Math. Sci., Developments of Cartan Geometry and Related Mathematical Problems (ed. T. Morimoto).

[ 9 ] T. Kobayashi, Propagation of multiplicity-free property for holomorphic vector bundles, math.RT/0607004. (Preprint).

[10] T. Kobayashi, A generalized Cartan decomposition for the double coset space $\left(U\left(n_{1}\right) \times U\left(n_{2}\right) \times\right.$ $\left.U\left(n_{3}\right)\right) \backslash U(n) /(U(p) \times U(q))$, J. Math. Soc. Japan, 59 (2007), 669-691, math.RT/0607006. (to appear).

[11] T. Kobayashi, Visible actions on symmetric spaces, Transformation Groups, math.DG/ 0607005. (to appear). 